be directly determined. Messrs. Bellingham and Stanley, L.td., also exhibited quartz spectrographs and a range of polarimeters and spectrometers, including a compact instrument for the examination of the entire spectrum giving readings directly in wave-lengths.

The ophthalmic section of the industry was well represented by about twenty-five different exhibitors. Many new types of spectacle lenses, eyeglass mounts and spectacle frames were shown. The ophthalmic instruments on view illustrated the development of apparatus for the detection of pathological conditions of the eye, as well as those for the mechanical measurement of visual errors. Among these may be mentioned the self-registering perimeters exhibited by several firms; the photographic ophthalmoscope, exhibited by the Cambridge Instrument Co., Ltd., by means of which it is possible to obtain photographs of the fundus showing both the optic disk and the macula lutea on one plate; and the combined corneal microscope and slit lamp, exhibited by Messrs. Clement Clarke, Ltd., in which both the illuminating system and the microscope are mounted on an arc which has its centre of curvature at the focus of the illuminating beam.

It is, of course, quite impossible in an article of reasonable length to give anything approaching a full list of the various interesting pieces of apparatus on view and described in the 320-page catalogue prepared for the use of the members of the Convention. In the preceding paragraphs reference has been made to only a few of the items on the stands of a few of the trade exhibitors, and many important items in important groups of instruments have had to be omitted.

The educative value of the exhibition was enhanced by the inclusion of a section devoted to optical instruments and apparatus designed by research workers for use in scientific investigations. These included an instructive collection of apparatus exhibited by the National Physical Laboratory, Teddington; the Technical Optics Department of the Imperial College of Science and Technology, South Kensington; the Department of Applied Optics, Northampton Polytechnic Institute, London; the Refraction Hospital, London; and by other institutions and scientific workers. Mention should be made of the selenium apparatus designed by Dr. E. E. Fournier d'Albe and E. O. Symonds for registering mechanically the passage of interference fringes ; the Clarendon photoelectric photometer, exhibited by Prof. F. A. Lindemann and Dr. G. M. B. Dobson, by means of which very small areas, such as spectral lines on a photographic plate, can be measured to a high degree of accuracy; and the series of instruments exhibited by the Cambridge Instrument Co., Ltd., in which the stylus-on-celluoid method is used for recording various physical phenomena.

The historical section of the exhibition contained a valuable loan collection of more than 350 items of great historical importance, illustrating the evolution and development of various types of optical instruments and indicating the types actually made and in use at different periods. Our admiration for the many highly developed and finished products of modern science in the adjoining sections did not in any way detract from our admiration for these optical treasures of past generations; for did not each one represent a modest though none the less distinctive landmark along the path of optical progress? The magnificent and impressive display of present-day apparatus, instruments and methods which formed the Optical Convention 1926 Exhibition marks the farthest point yet reached along that path.

\title{
The Intermittent Theory of Radiation.
}

$\mathrm{A}^{\mathrm{N}}$ important paper on "The Intermittence of to Electric Force" was read by Sir J. J. Thomson We now know that the atom is made up of electrons and positive charges, and that the number of electrons in the atom of an element is equal to the atomic number of the element. We also know the masses of the electrons and the positive charges. If, therefore, they obeyed the ordinary laws of electrostatics, we could calculate by the laws of dynamics the behaviour of the atom. The behaviour predicted in this way is not in accord with experiment. The reason of this discrepancy is doubtless that in the atom we are concerned with effects which occur in times and in distances very much shorter than those which occurred in the experiments by which the laws of electric force were discovered or tested.

Sir Joseph directs attention to the possibility of the electric force being intermittent in its action. The intermittence is so rapid that its effects are only apparent when we are dealing with events which occur in intervals as short as those in the atom. He illustrates this point by considering the properties of gases. So long as we test these properties on an ' engineering scale' they are completely explained by supposing that the gas exerts a continuous pressure on the walls of the containing vessel and that it obeys the hydrodynamical laws for a continuous fluid. It is known, however, that the pressure is not really continuous but consists of a succession of isolated blows following each other irregularly at very small intervals of time. By the methods now available for producing very high vacua we can get the gas in a state where it is impossible to explain its be- haviour by the hypothesis of continuity. The gas has a structure in time, the fineness of which is determined by the interval between the collisions. The fineness of the structure for a given gas varies with the pressure, being coarse at low pressure and fine at high pressure.

Sir Joseph supposes that the electric field has a structure in time, so that instead of the electric force being a continuous effect consisting of a continuous flow of momentum into the body under its action, it is really discontinuous and consists of finite increments of momentum separated by finite intervals of time. This discontinuity in the nature of the force readily explains the occasional spontaneous dissociation of a system. It also gives a reasonable hypothesis to explain why a system sometimes radiates away its surplus energy as Röntgen rays.

The assumption of the intermittence of electric force leads to startling results when applied to light waves. The mathematical proofs given indicate that there can be no unlimited propagation of light waves diverging from a source. The waves must ultimately reach a region where they are reflected back.

It will thus be seen that the hypothesis demands a corpuscular theory of light. The energy of the light must be done up into batches which retain their energy undiminished as they travel out into space. This does not exclude, in fact it requires, the presence of electrical waves of Maxwell's type. We must picture the light unit as a central quantum vibrating with the period of the light and emitting electrical waves. None, however, is allowed to escape, and the energy lost by the quantum in radiation is restored to it.

$$
\text { NO. 2947, VOL. I I 7] }
$$


Light, therefore, has a dual character. It consists of electrical waves and the quantum. The electrical waves give rise to interference effects and the quantum to photo-electric effects. The energy in the electrical waves surrounding the quantum does not alter as the system travels through space, and so the ratio of the energy in these waves and that in the quantum remain invariable. The mathematical proofs based on the theory of probability given in this paper are very neat and very easy to understand.

In a paper read before the Royal Society of Edinburgh on February 8, Prof. E. T. Whittaker follows up Sir J. J. Thomson's paper and shows that the Maxwell-Lorentz equations can easily be modified so as to represent Sir Joseph's ideas regarding light. What distinguishes Thomson's theory from the work of all other writers on the subject is that he pictures the structure of the light-quanta in terms of tubes of electric force. When light is generated he invites us to picture a closed part of a loop in a tube of electric force to become detached and go off as a closed ring. This ring soon becomes circular and travels with the velocity of light in a direction at right angles to its plane, like a circular vortex ring. The energy of the ring remains constant until the ring is broken.
It is known, for example, that if cathode rays fall on a metallic plate and generate $\mathrm{X}$-rays which fall on a second metallic plate, electrons are ejected from the second plate and their energy is of the same order as that of the electrons in the primary cathode rays and is independent of the distance of the plates.

The only explanation seems to be that the energy of the X-rays is contained in compact parcels between the plates. This phenomenon has led Einstein and other physicists to advocate corpuscular theories of light. In Thomson's theory we picture something similar to a procession of rings of cigarette smoke, moving forward through the air in front of the mouth of a smoker. As the group moves through space, it sweeps over the interior of an infinitely long, straight cylinder, the axis of which is in the direction of propagation. Everywhere outside this cylinder the field is permanently null.

Sir Joseph points out that it is not necessary to suppose that light consists exclusively of a procession of 'electric vortex rings.' $\mathrm{He}$ considers that it consists of rings of electric force accompanied by Maxwellian waves. These rings sometimes vibrate and produce waves of the type ordinarily considered. Whittaker gives a justification of Thomson's hypothesis and a verification of the quantum relation.

\section{Salient Features in the Stratigraphy, Tectonic Structure, and Physiography of the Commonwealth of Australia. ${ }^{1}$}

A BRIEF summary of the salient features of the stratigraphy of the Commonwealth is essential for understanding its tectonic structure. Pre-Cambrian rocks are developed in Western, Central, and South Australia. The Proterozoic rocks of Northern Australia are partly formed of thick dolomites, possibly a forerunner of the Great Barrier Reef of to-day. Basic lavas and tuffs, 3000 feet thick, preceded a great ice-age in late Proterozoic or early Cambrian time. Marine Cambrian strata occur chiefly in Northern and Central Australia and South Australia, with patches in Victoria, Tasmania, and Queensland. Ordovician rocks attain a thickness of 40,000 feet in Victoria and Central Australia. Igneous intrusions and unconformity separate the Ordovician rocks from the Silurian. In the Devonian rocks of Queensland, coralline limestone, 7000 feet thick, is probably another ancestor of the Great Barrier Reef. In Carboniferous time a south-eastern shore of 'the Tethys Sea ' is well defined in the northwest of Western Australia. Carboniferous and Devonian time were periods of granitic intrusions and effusive rocks. In Permo-Carboniferous time half Australia was under ice. This was the chief coal-forming epoch, followed by more coal (in Triassic and in Jurassic times) developed in immense epicontinental lakes in eastern and central Australia, near which carnivorous deinosaurs existed.

Marine Jurassic strata in Australia are only found in 'the Tethys Sea' of Western Australia. The great dolerite-sills (comparable with the Karroo dolerites) of Tasmania possibly closed Jurassic time. Cretaceous time witnessed great peripheral and central marine transgressions. The Neocomian Sea was probably cold, and epeirogenic uplift in east Central Australia replaced marine by lacustrine conditions; this uplift was probably accompanied by small local glaciation. In Western Australia the last of "the 'Tethys Sea' is represented by the Lower Santonian with Uintacrinus (comparable with the Arriloor Beds of Southern India). Cretaceous strata were slightly

I Substance of a lecture delivered by Sir T. W. Edgeworth David, K.B.E. F.R.S., before the Geological Society of London on March 29. folded in pre-Oligocene time. Eocene marine deposits are mostly wanting except, perhaps, in New Guinea, but the Winton series may be partly Eocene.

Oligocene time was one of very extensive peneplanation, which was prolonged into the Miocene, and in places into early Pliocene time. A rock-crust, or 'petroderm' ('Hartrinde' of Walther) formed over the more arid parts of this peneplain. Marine transgressions occurred all along the southern coast of Australia, mostly following on the eruptions of the older basalts.'

In Miocene time there were warm seas with large foraminifera (Lepidocyclina) and reef-forming corals, even in Tasmania. The earliest marsupials are found there in the Miocene beds. Miocene browncoal deposits up to 800 feet thick, formed partly of Fagus and Banksia, occur in Victoria. 'Deep leads' of gold and tin were formed inland. In New Guinea the oil-belt was developed during the Miocene Period.

In Pliocene time the whole peneplain underwent differential movement which was strongest in eastern Australia. Alkaline lavas were erupted from tensional fault-planes in the old peneplain. Epeirogenic uplift (during the 'Kosciusko epoch' of E. C. Andrews) now followed, and became accelerated in a late Pliocene epoch. The Rift Valley of Australia formed mostly during this uplift. This later movement was accompanied by widespread eruptions of basalts with some andesites. The Great Barrier Reef was already forming, and possibly commenced to develop even earlier.

The southern edge of the Lake Eyre peneplain became tilted northwards, and that of Western Australia eastwards, bringing about interior drainage and local aridity. The Pleistocene ice-age followed, together with a pluvial epoch, when Lake Eyre once more drained seawards. Crocodiles and gigantic marsupials were then abundant in Central Australia. The Tasmanian aborigines arrived in Tasmania by way of the Malay Bridge, possibly during a eustatic negative movement of the sea. As the Ice Age ended, the sea-level rose, drowning the coastal valleys. The rainfall decreased. Lastly, the Australian aborigines 